\title{
Endogenously increased $n-3$ PUFA levels in fat-1 transgenic mice do not protect from non-alcoholic steatohepatitis
}

\author{
Marie Liebig ${ }^{1}$, Dirk Dannenberger ${ }^{2}$, Brigitte Vollmar $^{1}$, Kerstin Abshagen $^{1}$ \\ ${ }^{1}$ Rudolf-Zenker-Institute for Experimental Surgery, University Medicine Rostock, 18057 Rostock, Germany; ${ }^{2}$ Institute of Muscle Biology and \\ Growth, Leibniz Institute for Farm Animal Biology (FBN), 18196 Dummerstorf, Germany \\ Contributions: (I) Conception and design: K Abshagen, B Vollmar; (II) Administrative support: K Abshagen, B Vollmar; (III) Provision of study \\ material or patients: None; (IV) Collection and assembly of data: M Liebig; (V) Data analysis and interpretation: M Liebig, D Dannenberger, K \\ Abshagen; (VI) Manuscript writing: All authors; (VII) Final approval of manuscript: All authors. \\ Correspondence to: Kerstin Abshagen, PhD. Rudolf-Zenker-Institute for Experimental Surgery, University Medicine Rostock, Schillingallee 69a, 18057 \\ Rostock, Germany. Email: kerstin.abshagen@uni-rostock.de.
}

\begin{abstract}
Background: Non-alcoholic fatty liver disease (NAFLD) is the most common chronic liver disease worldwide, ranging from simple steatosis to non-alcoholic steatohepatitis (NASH) and fibrosis. Possible reasons for the NAFLD epidemic in industrialized countries are the high intake of pro-inflammatory n-6 polyunsaturated fatty acids (n-6 PUFAs) and low consumption of healthy n-3 PUFAs. Due to their antiinflammatory properties, n-3 PUFAs may have the potential to alleviate chronic liver disease. Herein, we examined the therapeutic effect of increased n-3 PUFA tissue levels in fat- 1 transgenic mice on progressive NASH.
\end{abstract}

Methods: Disease was induced in mice by streptozotocin and high fat diet (STZ/HFD) resulting in NASH. NAFLD in 6 and 8 weeks old wild type and fat- 1 transgenic STZ/HFD treated mice was analyzed. Unlike all other mammals, fat- 1 transgenic mice ubiquitously express an $n-3$ fatty acid desaturase, which converts n- 6 to n-3 PUFAs, leading to increased n-3 and decreased n-6 PUFA tissue contents.

Results: Liver damage, NAFLD activity score (NAS), hepatic lipid accumulation and inflammation were significantly reduced in fat- 1 transgenic STZ/HFD treated mice in the early (6 weeks) but not late ( 8 weeks) phase of NASH. Simultaneously, mRNA expression of genes involved in fatty acid uptake and storage ( $C d 36$ and Plin3, respectively) was significantly down-regulated in 6 week old but not 8 week old fat- 1 transgenic STZ/HFD treated mice.

Conclusions: Endogenously elevated n-3 PUFA levels in fat-1 transgenic mice transiently delay the onset of STZ/HFD induced NASH but failed to efficiently protect from NASH development.

Keywords: Non-alcoholic fatty liver disease (NAFLD); non-alcoholic steatohepatitis (NASH); n-6/n-3; fat-1; steatosis

Submitted Nov 28, 2018. Accepted for publication Feb 26, 2019.

doi: $10.21037 / \mathrm{hbsn} .2019 .04 .03$

View this article at: http://dx.doi.org/10.21037/hbsn.2019.04.03

\section{Introduction}

Affecting approximately $25 \%$ of the world's population, non-alcoholic fatty liver disease (NAFLD) is the most common chronic liver disease worldwide (1) and is closely linked to obesity and metabolic syndrome. Due to its rapidly increasing prevalence, a $178 \%$ rise in NAFLD related liver deaths by 2030 is estimated (2). NAFLD ranges from "simple" steatosis to non-alcoholic steatohepatitis (NASH) which elevates the risk for progression to cirrhosis and hepatocellular carcinoma (3). Hence, NAFLD is becoming an increasingly threatening disease and understanding its progression is of great interest.

Over the past decades, high dietary uptake of pro- 
inflammatory n-6 polyunsaturated fatty acids (n-6 PUFAs) and insufficient supply with salutary n-3 PUFAs in industrialized countries have led to a disadvantageous dietary n-3/n-6 PUFA ratio in favor of n-6 PUFAs (4). Numerous positive and protective effects, e.g., regarding tumorigenesis, diabetes, obesity and cardiovascular disease, were attributed to n-3 PUFAs (5). Furthermore, n-3 PUFAs have been shown to alleviate hepatic steatosis (6). Conversely, n-6 PUFAs induce platelet aggregation, vasoconstriction and inflammation (7). It is well known that the effects of PUFAs are mediated by bioactive lipid mediators such as resolvins and prostaglandins $(7,8)$. As their synthesis from n-3 and n-6 PUFAs relies on the same converting enzymes $(9,10)$, the n-3/n-6 PUFA ratio is crucial for the overall effect and has been hypothesized to be essential for the positive effects of n-3 PUFAs. Due to their anti-inflammatory and anti-steatotic properties, increasing endogenous n-3 PUFAs as well as the $n-3 / n-6$ PUFA ratio might serve as effective therapeutic strategies alleviating NAFLD and NASH.

To date, apart from life style modification, no therapeutic interventions against NAFLD exist. Therefore, it is of utmost importance to understand NAFLD. Herein, we report that fat- 1 transgenic mice with endogenously increased n-3 PUFA levels and n-3/n-6 PUFA ratios are not protected from NASH development despite a delayed onset of the disease.

\section{Methods}

\section{Mice}

Fat-1 transgenic breeder mice (11) with C57BL/6J background were provided by Dr. Weylandt (Charité University Medicine, Berlin, Germany) with kind permission of Dr. Kang (Massachusetts General Hospital and Harvard Medical School, Boston, Massachusetts, USA). Fat- 1 transgenic mice express the $\mathrm{n}-3$ fatty acid desaturase (fat-1) gene from C. elegans which converts n-6 PUFAs to n-3 PUFAs (11). This leads to increased n-3 PUFA tissue levels and an increased n-3/n-6 PUFA ratio $(12,13)$. The presence of the fat 1 gene was confirmed by genotyping of each mouse (primer: forward 5'-CTTCACCATGCTTTCACCAACC-3' reverse 5'-AC ACAGCAACAGATTCCAGAGATT-3'). Fat-1 transgenic (fat-1) and wildtype (wt) mice were housed on a $12 \mathrm{~h} / 12 \mathrm{~h}$ light/dark cycle with ad libitum access to water and food. All animal experiments were approved by "Landesamt für Landwirtschaft, Lebensmittelsicherheit und Fischerei Mecklenburg-Vorpommern" (7221.3-1-022/15) and were executed in accordance with the German legislation and EU-directive 2010/63/EU.

\section{Mouse model}

NASH was induced by streptozotocin and high fat diet (STZ/HFD) (14) as described previously by our group $(15,16)$. In short, male fat- 1 transgenic and wt mice were injected with $200 \mu \mathrm{g}$ streptozotocin (Sigma-Aldrich, St. Louis, Missouri, USA) in $10 \mu \mathrm{L}$ of $0.05 \mathrm{M}$ trisodium citrate ( $\mathrm{pH}$ 4.5) (Merck, Darmstadt, Germany) intraperitoneally at day two postnatal. From 28 days of age the mice were fed a continuous HFD [fat content: $60 \mathrm{~kJ} \%$; D12492(II) modified experimental diet; Ssniff, Soest, Germany]. The fatty acid composition of the diet is displayed in Table 1 . The mice develop NAFLD with progression from NASH to liver tumors within 20 weeks. To study the early phase of progressive NAFLD, blood and tissue was collected at the age of 6 and 8 weeks as described previously by our group (15). 14 wt mice and 11 fat- 1 transgenic mice at an age of 6 weeks as well as $10 \mathrm{wt}$ and 11 fat- 1 transgenic 8 week old mice were sacrificed. In case of only a subset of samples being analyzed, the exact number of analyzed samples per group is indicated in the methods section in parentheses behind the corresponding parameter in the following order: wt 6 weeks, fat- 16 weeks, wt 8 weeks, fat-1 8 weeks. The general state of health was monitored daily and blood glucose levels and body weight were measured weekly. Animals exhibiting normal blood glucose levels were excluded from the experiment.

\section{Plasma analyses}

Alanine aminotransferase (ALT) and glutamate dehydrogenase (GLDH) activity in EDTA plasma (sample size: $14,11,7,11$ ) and plasma triglyceride contents (sample size: $11,11,7,11)$ were measured as described previously (15). Free fatty acids (sample size: 10, 10, 7, 10; ab65341, Abcam, Cambridge, UK) and total cholesterol (sample size: 11, 11, 7, 11; ab65390, Abcam, Cambridge, UK) assays were performed according to the manufacturer's instructions.

\section{Histology and immunobistochemistry}

Histological and immunohistochemical stainings were performed as described previously (15) and were analyzed in 
Table 1 Fatty acid composition (in \% of total fatty acids) of the livers of 6 and 8 week old STZ/HFD treated fat-1 transgenic and wt mice as well as of the HFD

\begin{tabular}{|c|c|c|c|c|c|}
\hline Fatty acids & \multicolumn{2}{|c|}{6 weeks } & \multicolumn{2}{|c|}{8 weeks } & High fat diet \\
\hline C18:2n-6 & 9.85 & 10.24 & 8.93 & 9.21 & 6.68 \\
\hline C18:3n-3 & 0.35 & $0.42^{*}$ & 0.31 & $0.52^{\star}$ & 0.92 \\
\hline C20:4n-6 & 4.74 & 4.6 & 4.44 & $2.62^{\star \star}$ & n.d. \\
\hline$C 22: 4 n-6$ & 0.23 & $0.09^{\star \star \star}$ & 0.22 & $0.07^{\star \star \star}$ & 0.04 \\
\hline C22:6n-3 & 4.33 & $8.28^{* \star}$ & 4.36 & $6.84^{\star \star \star}$ & n.d. \\
\hline Sum SFA & 36.06 & $34.48^{*}$ & 34.37 & 35.43 & 53.19 \\
\hline Sum MUFA & 41.43 & $37.95^{\star}$ & 44.58 & 41.62 & 38.81 \\
\hline Sum n-6 PUFA & 16.52 & 16.82 & 15.16 & 13.42 & 6.79 \\
\hline n-6/n-3 PUFA ratio & 2.92 & $1.62^{\star \star \star}$ & 2.78 & $1.48^{\star \star \star}$ & 7.22 \\
\hline
\end{tabular}

Group differences were tested by $t$-test within each time point. ${ }^{\star} \mathrm{P}<0.05 \mathrm{vs}$. wt, ${ }^{\star \star} \mathrm{P}<0.01 \mathrm{vs}$. wt, ${ }^{* \star *} \mathrm{P}<0.001 \mathrm{vs}$. wt. STZ/HFD, streptozotocin and high fat diet; SFA, saturated fatty acids; MUFA, monounsaturated fatty acids; PUFA, polyunsaturated fatty acids; n.d., not detectable.

a blinded manner. Formalin fixed liver tissue was embedded in paraffin and cut into $5 \mu \mathrm{m}$ thick sections. For assessment of NAFLD activity score (NAS), the sections were stained with hematoxylin and eosin (Merck, Darmstadt, Germany). NAS was determined as proposed by Kleiner et al. (17) and as described previously by our group (15). The score for each section was determined by three independent observers. Infiltration of granulocytes in liver tissue was assessed by staining for chloroacetate esterase (CAE) with Naphthol AS-D chloroacetate (Sigma-Aldrich, St. Louis, Missouri, USA) and counterstaining with hematoxylin. For quantification CAE positive cells were counted in 30 consecutive high power fields (HPF). Immunohistochemical staining against $\mathrm{F} 4 / 80$ was conducted as described previously by our group $(15,18)$. For analysis of the staining at least 30 consecutive photomicrographs were taken using a 40x objective. The red stained F4/80-positive area was quantified using the color threshold tool in ImageJ $1.47 \mathrm{v}$. Oil Red O staining of lipids in liver tissue was performed using $8 \mu \mathrm{m}$ thick paraformaldehyde (ChemCruz, Dallas, Texas, USA) fixed frozen tissue sections that were subsequently stained with Oil Red O (sample size: 14, 11; 9, 11; SigmaAldrich, St. Louis, Missouri, USA) and counterstained with hematoxylin. For analysis at least 10 photomicrographs were taken per section using a $20 \times$ objective. The red stained area was quantified using ImageJ $1.47 \mathrm{v}$.

\section{$q R T-P C R$}

Total RNA was isolated from snap frozen liver tissue lysates using the RNeasy Mini Kit (Qiagen, Hilden, Germany) with the RNase-Free DNase Set (Qiagen, Hilden, Germany) for on column digestion of genomic DNA according to the manufacturer's instructions. For reverse transcription of $2 \mu \mathrm{g}$ RNA to cDNA, the Oligo(dT) $)_{18}$ mRNA Primer (New England Biolabs GmbH, Ipswich, Massachusetts, USA) and SuperScript ${ }^{\mathrm{TM}}$ II Reverse Transcriptase (Invitrogen, ThermoFisher Scientific, Waltham, Massachusetts, USA) were used. Quantitative Real-Time PCR gene expression analysis for the detection of amplified dsDNA strands was performed with the Bio-Rad iQ5 system (Bio-Rad Laboratories, Hercules, California, USA) using the iTaq ${ }^{\mathrm{TM}}$ Universal SYBR ${ }^{\circledR}$ Green Supermix (Bio-Rad Laboratories, Hercules, California, USA). The primers used for amplification are listed in Table 2. The results were calculated using the $2^{-\Delta \Delta C t}$ method with normalization of the target gene expression levels to the housekeeping gene expression (Rps18) and to expression levels of a liver tissue pool obtained from healthy wt C57BL/6J mice. qRT-PCR was performed with randomly selected liver tissue samples 
Table 2 Primers used for qRT-PCR analysis of mouse liver tissue

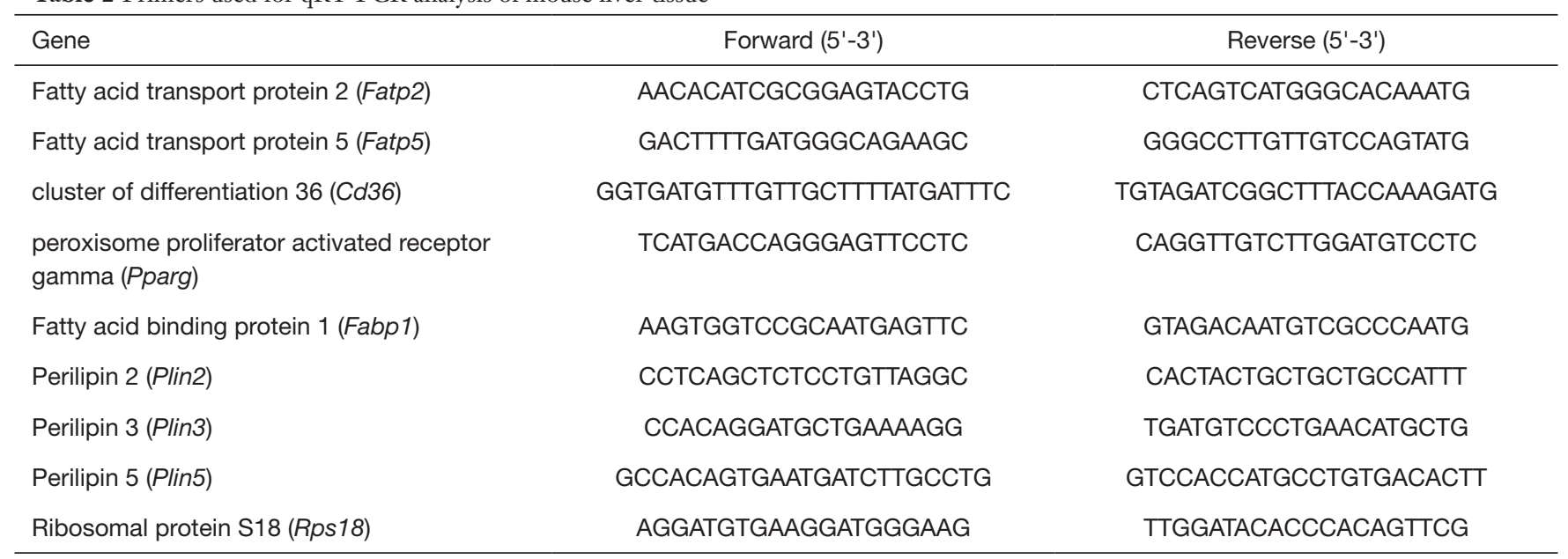

of 6 wt and 5 fat- 1 mice of each age.

\section{Lipid extraction and fatty acid analysis}

After homogenization of frozen liver samples and the addition of C19:0 as an internal standard, the total liver lipids were extracted in duplicate using chloroform/ methanol $(2: 1, \mathrm{v} / \mathrm{v})$ by the use of Ultra Turrax T25 (IKA, Staufen, Germany), $3 \times 15 \mathrm{sec}, 15,780 \times \mathrm{g}$, at room temperature. The detailed sample preparation procedure has been previously described (19). Briefly, the organic phase was separated and dried with $\mathrm{Na}_{2} \mathrm{SO}_{4}$ and $\mathrm{K}_{2} \mathrm{CO}_{3}$ $(10: 1, w / w)$, and the solvent was subsequently removed under gentle nitrogen at room temperature. Next, $2 \mathrm{~mL}$ of $0.5 \mathrm{M}$ sodium methoxide in methanol was added to the samples, which were shaken in a $60{ }^{\circ} \mathrm{C}$ water bath for $10 \mathrm{~min}$. Subsequently, $1 \mathrm{~mL}$ of $14 \%$ boron trifluoride in methanol was added to the mixture, which was then shaken for additional 10 minutes at $60{ }^{\circ} \mathrm{C}$. Saturated $\mathrm{NaHCO}_{3}$ solution $(2 \mathrm{~mL})$ was added, and the fatty acid methyl esters (FAMEs) were extracted three times in $2 \mathrm{~mL}$ of $\mathrm{n}$-hexane. The FAMEs were stored at $-18{ }^{\circ} \mathrm{C}$ until used for gas chromatography (GC) analysis. The fatty acid analysis of the liver lipids was performed using capillary GC with a CP-Sil $88 \mathrm{CB}$ column $(100 \mathrm{~m} \times 0.25 \mathrm{~mm}$, Agilent, Santa Clara, CA, United States) that was installed in a PerkinElmer gas chromatograph CLARUS 680 with a flame ionization detector and split injection (PerkinElmer Instruments, Shelton, United States). The detailed GC conditions were recently described (20). Briefly, hydrogen was used as the carrier gas at a flow rate of $1 \mathrm{~mL} \mathrm{~min}{ }^{-1}$. The split ratio was 1:20, and the injector and detector were set at 260 and $280^{\circ} \mathrm{C}$, respectively. The quantification of fatty acids was done by the use of C19:0 as internal standard. For the calibration procedure the reference standard mixture "Sigma FAME" (Sigma-Aldrich, Deisenhofen, Germany), the methyl ester of C18:1cis-11, C22:5n-3 and C18:2cis-9, trans-11 (Matreya, State College, PA, USA), C22:4n-6 (SigmaAldrich, Deisenhofen, Germany) and C18:4n-3 (Larodan, Limhamn, Sweden) were used. The five-point calibration of single fatty acids ranged between 16 and $415 \mathrm{mg} / \mathrm{mL}$ and was checked after GC analysis of five samples.

\section{Statistical analysis}

Statistical analyses were performed using GraphPad Prism 6.05 (GraphPad Software, La Jolla, California, USA). The group difference within each time point was assessed by $t$-test and is indicated in the graphs as follows: ${ }^{*} \mathrm{P}<0.05$, ${ }^{* *} \mathrm{P}<0.01,{ }^{* *} \mathrm{P}<0.001$. Statistical significance was set at $\mathrm{P}<0.05$. All data are presented as mean \pm standard deviation $(\mathrm{SD})$ in dot plots.

\section{Results}

\section{General aspects}

Liver fatty acid analysis revealed significantly increased hepatic contents of n-3 PUFAs and significantly decreased n-6/n-3 PUFA ratios in fat- 1 transgenic STZ/HFD mice compared to wt at both time points. Compared to fat-1 transgenic STZ/HFD mice, livers of wt STZ/HFD mice 
A
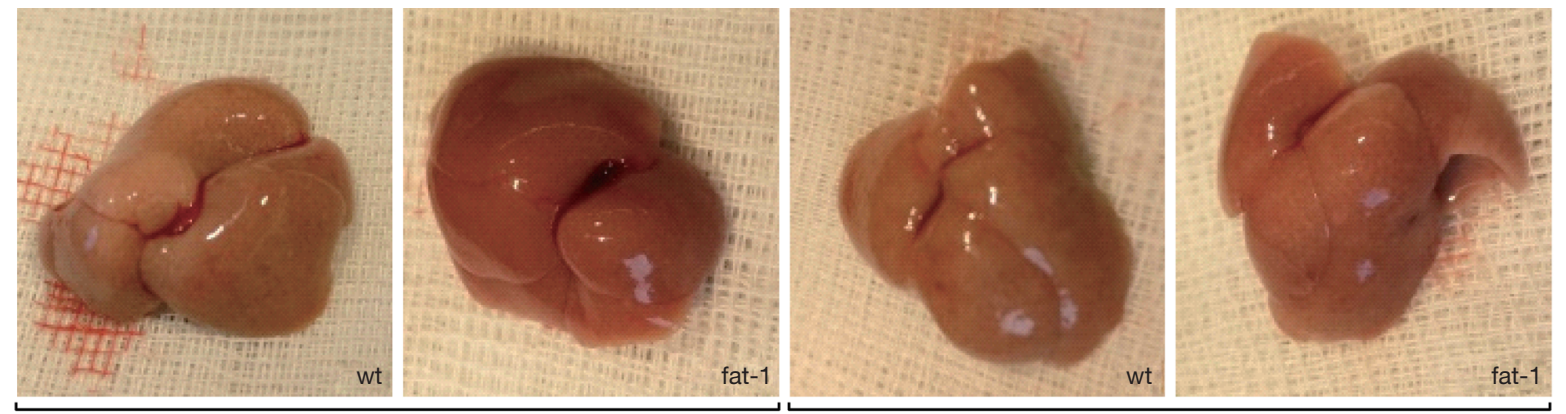

6 wks

8 wks

B

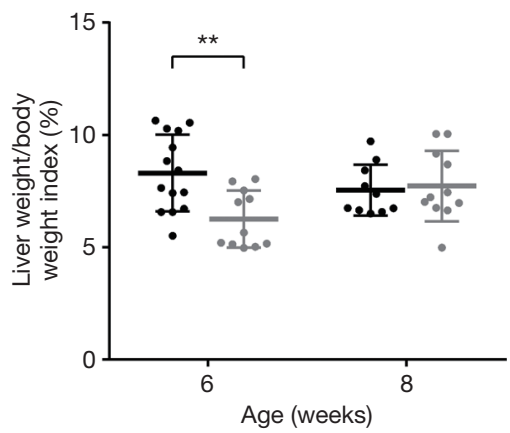

E

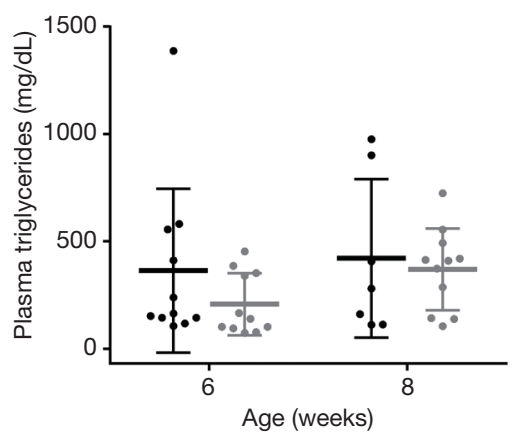

C

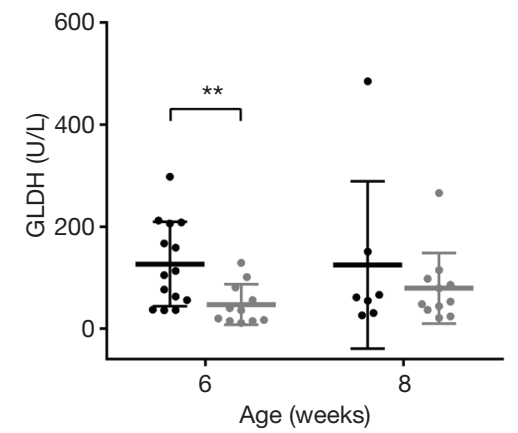

F

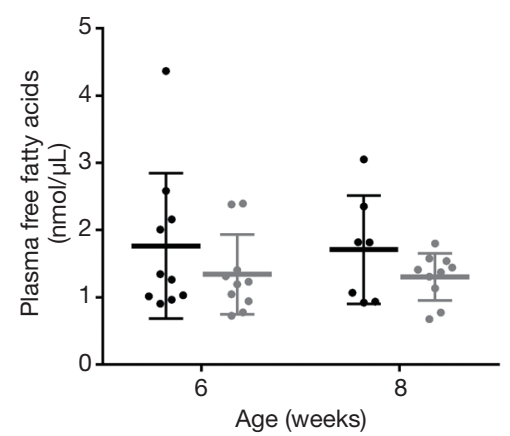

D

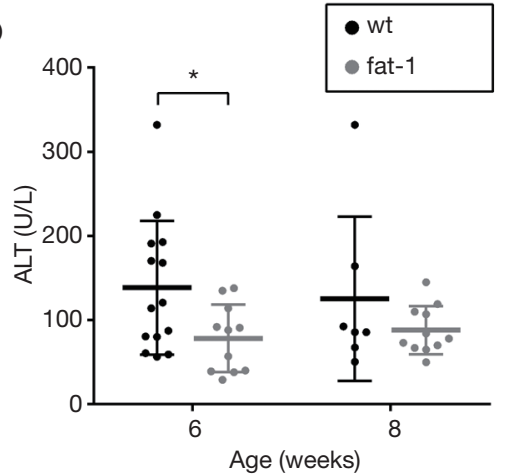

G

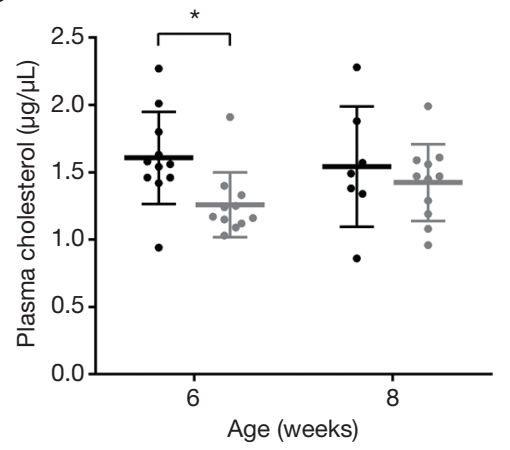

Figure 1 Representative images of livers of 6 and 8 weeks old STZ/HFD treated wt and fat-1 mice (A) and analysis of liver weight/body weight index (B), plasma activity of GLDH (C) and ALT (D) as well as plasma content of triglycerides (E), free fatty acids (F) and total cholesterol (G) of 6 and 8 week old STZ/HFD treated wt and fat- 1 mice. Values are presented as mean \pm SD. Group differences were tested by $t$-test within each time point. ${ }^{*} \mathrm{P}<0.05,{ }^{* *} \mathrm{P}<0.01, \mathrm{n}=7-14$.

appeared palish in color, particularly at 6 weeks (Figure 1A). Moreover, the liver weight/body weight index was significantly reduced by $2 \%$ in fat- 1 transgenic STZ/HFD mice compared to wt STZ/HFD mice at 6 weeks but not at 8 weeks of age (Figure 1B). Liver damage, as assessed by ALT and GLDH activities in plasma, was transiently alleviated in fat-1 compared to wt STZ/HFD mice (Figure 1C,D), showing significantly reduced levels at 6 weeks of age. Plasma triglyceride (Figure 1E), free fatty acid (Figure 1F) and total cholesterol levels (Figure 1G) trended to be reduced in fat- 1 compared to wt STZ/HFD mice. This reduction was more pronounced at 6 weeks of age and was significant for plasma cholesterol at this time point, but less prominent at 8 weeks of age. Despite these differences, blood glucose levels did not differ between the groups (data not shown).

\section{Histopathology}

The NAS was determined for histopathologic evaluation of 
A

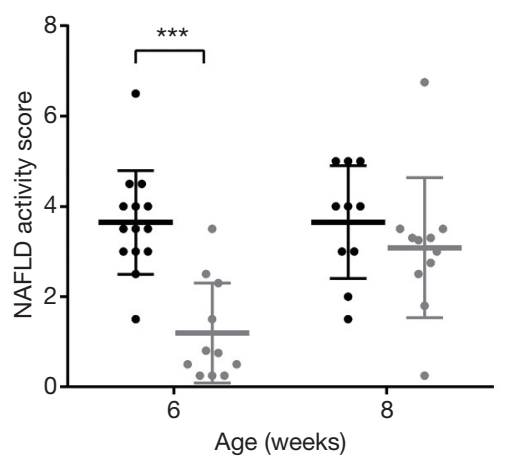

C

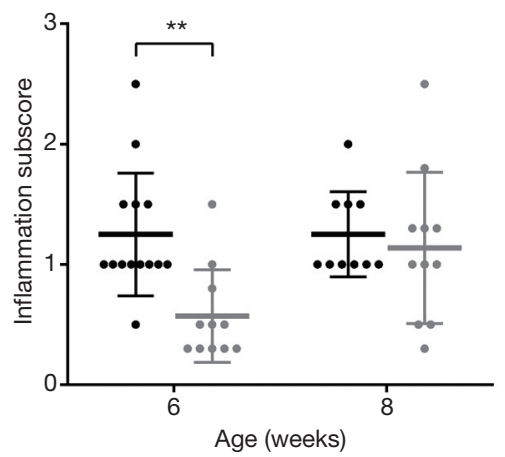

B

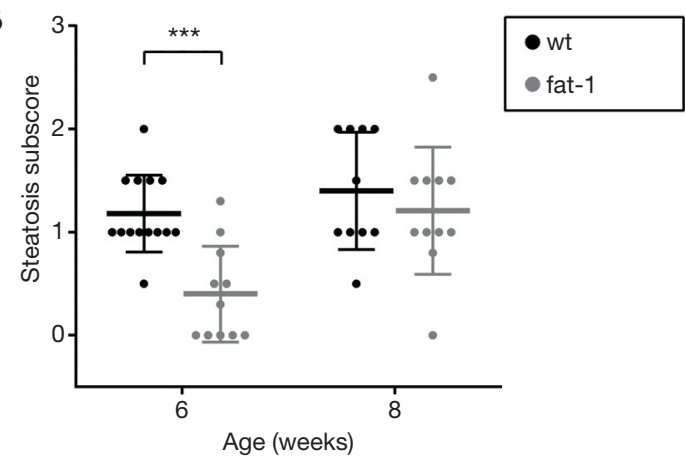

D

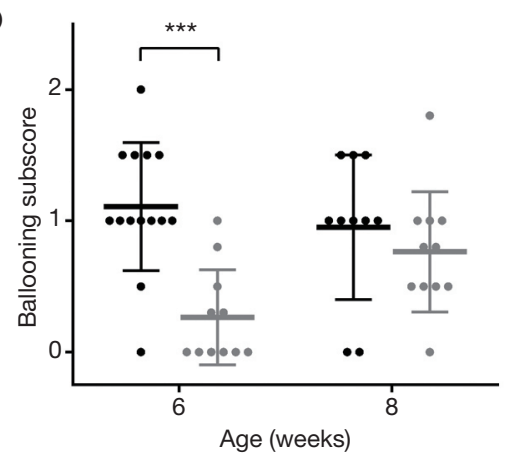

Figure 2 NAFLD activity score (A) and its subscores for steatosis (B), inflammation (C) and ballooning (D) of 6 and 8 week old STZ/HFD treated wt and fat- 1 mice. Values are presented as mean $\pm \mathrm{SD}$. Group differences were tested by $t$-test within each time point. ${ }^{* *} \mathrm{P}<0.01$, ${ }^{* * *} \mathrm{P}<0.001, \mathrm{n}=10-14$ per group.

NAFLD (Figure 2A). Interestingly, fat-1 STZ/HFD mice exhibited a highly significantly lower NAS than wt STZ/ HFD mice at 6 weeks of age (wt: 3.6, fat-1: 1.2), whereas no difference was detected at an age of 8 weeks. The same trend was observed for all subscores (steatosis, inflammation and ballooning; Figure 2B,C,D, respectively), with the reduction in fat-1 STZ/HFD mice being highly significant at 6 weeks of age.

For further characterization of hepatic steatosis (Figure 3), Oil Red $\mathrm{O}$ stained liver sections were analyzed quantitatively (Figure $3 A, D$ upper panel). While wt STZ/HFD mice revealed massive accumulation of hepatic fat, Oil Red O-positive area was markedly reduced in livers of fat-1 STZ/ HFD mice. The difference was highly significant at 6 weeks of age, where in fat-1 STZ/HFD mice only $~ 13.5 \%$ of the liver was Oil Red O-positive compared to $40 \%$ in wt STZ/ HFD mice. At an age of 8 weeks the difference was less pronounced and did not reach statistical significance.

As inflammation is a key feature of NASH, immigrated granulocytes were assessed by CAE staining (Figure 3B,D lower panel). Interestingly, significantly fewer $\mathrm{CAE}$ positive cells per HPF were observed in fat- 1 compared to wt STZ/ HFD mice at the age of 6 weeks, whereas this difference disappeared at 8 weeks. Furthermore, we investigated the abundance of hepatic macrophages by F4/80 immunohistochemistry (Figure 3C). However, no difference between fat-1 and wt STZ/HFD mice was observed.

\section{Fatty acid transport and storage}

Hepatic mRNA expression of fatty acid transport protein (Fatp) 2 (Figure 4A) and 5 (Figure 4B) and fatty acid binding protein Fabp1 (Figure $4 C$ ) did not differ between the genotypes. Nevertheless, the STZ/HFD-induced increase of hepatic $C d 36$ mRNA expression of wt mice was significantly lower in fat- 1 transgenic mice at 6 weeks of age (Figure $4 D$ ). However, no difference was detectable at 8 weeks of age. CD36 is a scavenger receptor involved in lipid signaling, uptake and transport. Its mRNA expression is known to be regulated by PPAR- $\gamma$. Analysis of hepatic Pparg mRNA expression levels revealed significantly decreased values for fat-1 STZ/HFD mice at both time points (Figure 4E). 

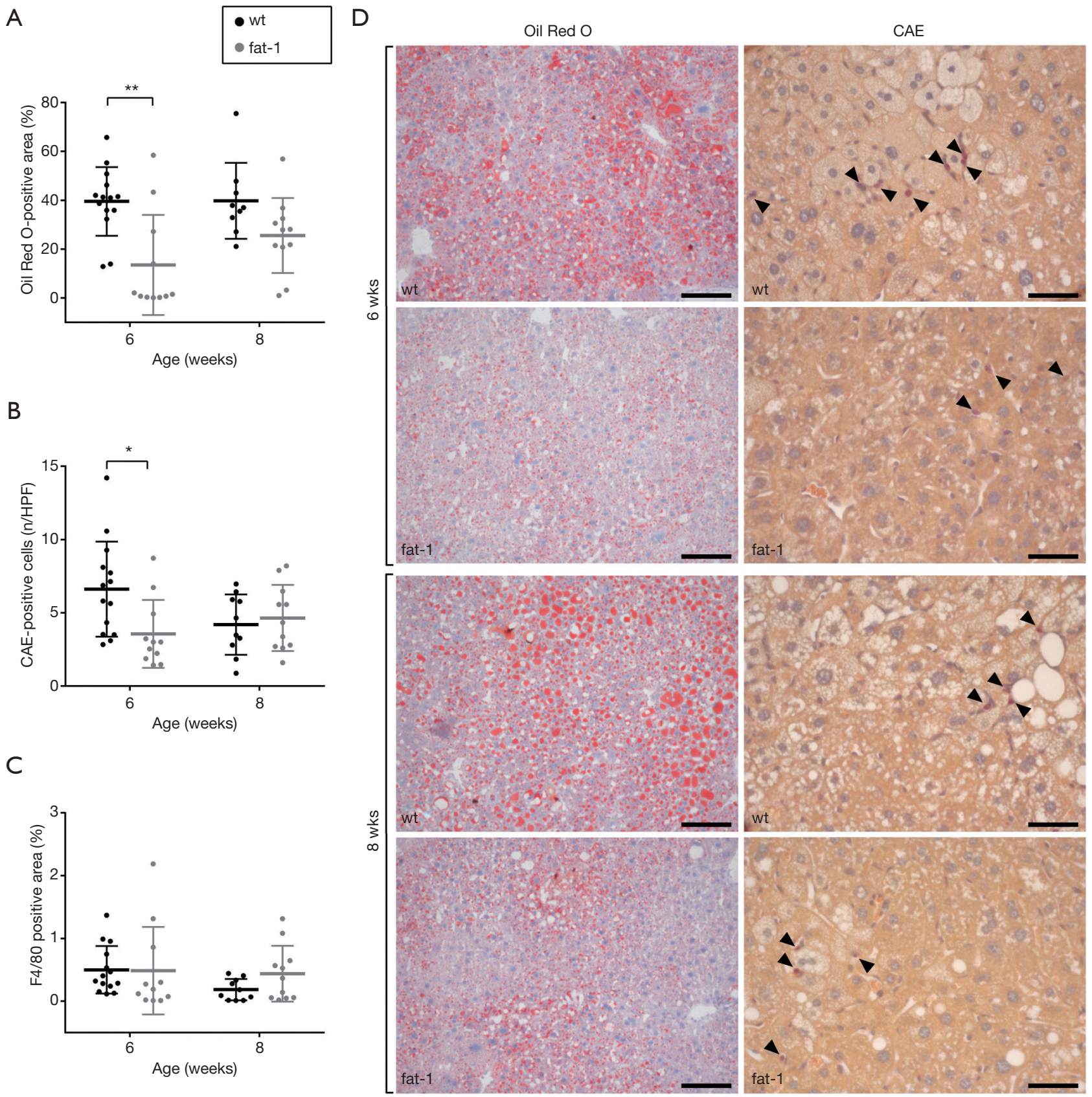

C

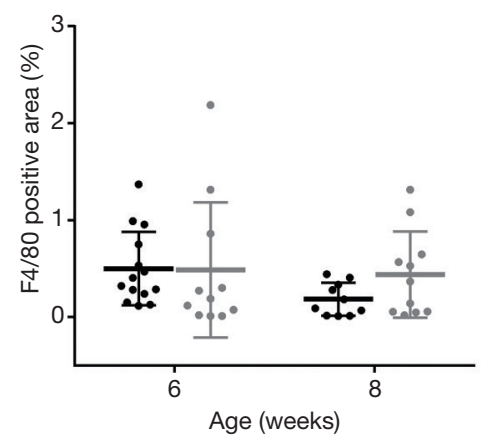

Figure 3 Quantitative analysis of Oil Red O (A), CAE (B) and F4/80 (C) stained liver sections of 6 and 8 week old STZ/HFD treated wt and fat 1 mice. Values are presented as mean $\pm \mathrm{SD}$. Group differences were tested by $t$-test within each time point. ${ }^{*} \mathrm{P}<0.05,{ }^{* *} \mathrm{P}<0.01, \mathrm{n}=9-14$ per group. (D) Representative photomicrographs of Oil Red O (left panel) and CAE (right panel) stained liver sections of 6 and 8 week old STZ/HFD treated wt and fat-1 mice. The scale bars mark $100 \mu \mathrm{m}$ (Oil Red O) and $50 \mu \mathrm{m}$ (CAE), respectively. CAE positive cells are marked by arrowheads.

NAFLD is known to be aggravated by the perilipin (Plin) protein family. As Plins play a role as structural proteins on lipid droplets, we analyzed mRNA expression of Plin2
(Figure 4F), Plin3 (Figure 4G) and Plin5 (Figure 4H). Compared to wt STZ/HFD mice, 6 week old fat-1 transgenic STZ/HFD mice exhibited reduced Plin 2 and 

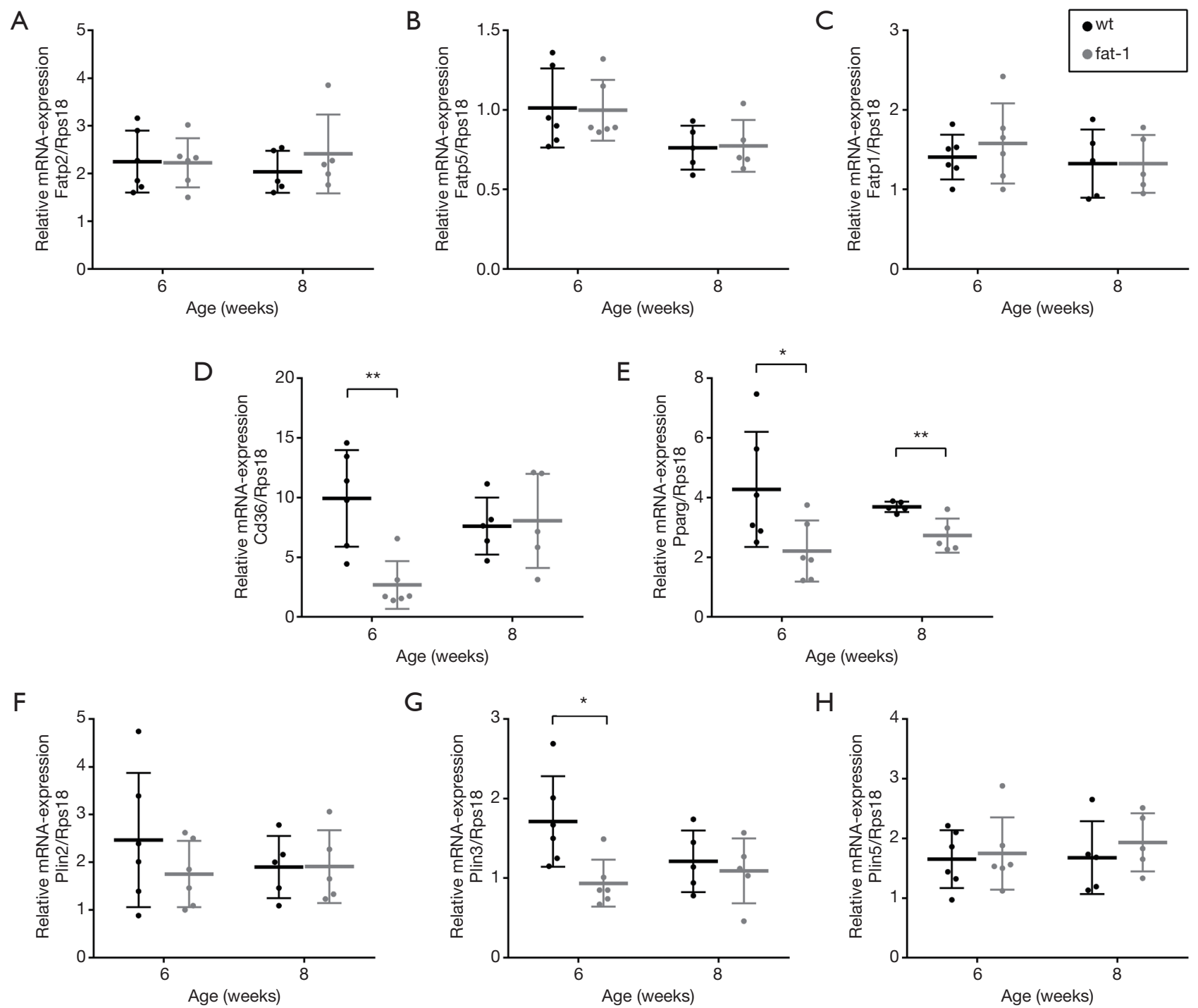

Figure 4 mRNA expression of Fatp2 (A), Fatp5 (B), Fabp1 (C), Cd36 (D), Pparg (E), Plin2 (F), Plin3 (G) and Plin 5 (H) relative to Rps18 in liver tissue of 6 and 8 week old STZ/HFD treated wt and fat-1 mice. Values are presented as mean \pm SD. Group differences were tested by $t$-test within each time point, ${ }^{*} \mathrm{P}<0.05,{ }^{* *} \mathrm{P}<0.01$. $\mathrm{n}=5-6$ per group.

Plin3 mRNA expression levels, which was significant for Plin3. However, at 8 weeks of age both genotypes showed similar mRNA expression levels. Interestingly, Plin 5 mRNA expression did not differ between the groups at both time points.

\section{Discussion}

Herein, we reported that endogenously elevated n-3 PUFA tissue contents in fat- 1 transgenic mice may delay the onset of experimental NASH, but do not impede NASH development. While a positive effect of n-3 PUFAs on steatosis and NAFLD in general has been confirmed by numerous studies $(21,22)$, the effect of n-3 PUFAs on NASH is discussed controversially. In accordance with our findings, a double-blind, randomized, placebo-controlled trial in NASH patients showed no impact of n-3 PUFA supplementation on liver injury, despite the liver fat content being significantly reduced (23). In another clinical trial, synthetic eicosapentaenoic acid (C20:5n-3) was unable to alleviate NASH pathology but reduced plasma triglyceride levels (24). Provenzano et al. even observed worsening of 
NASH regarding inflammation and liver damage in $n-3$ PUFA supplemented methionine choline-deficient diet fed mice (25). These findings indicate that dietary n-3 PUFAs have a limited therapeutic potential to ameliorate NASH. However, the observed effects of n-3 PUFAs depend on multiple factors including dietary composition, n-3 PUFA tissue availability as well as liver disease model and thus, the inflammatory state. Most studies examining n-3 PUFA supplementation do not evaluate the overall n-3/n-6 PUFA ratio of the diet or tissue, which might significantly affect the outcome of the studies. Thus, studies using fat-1 transgenic mice, which diet-independently exhibit elevated tissue n-3 PUFA levels and n-3/n-6 PUFA ratio, are interesting as they allow linking the observed effects to the endogenous availability of n-3 PUFAs. Several studies that have examined HFD fed fat- 1 transgenic mice, observed, i.e., reduced steatosis, ballooning, fibrosis, ALT, serum and liver triglycerides and total cholesterol $(26,27)$. Additionally, in another disease model fat-1 transgenic mice seem to be protected from high fat plus high sugar diet induced NAFLD, exhibiting reduced steatosis compared to wt mice (28). Although we observed transiently alleviated NAFLD in fat- 1 mice, n-3 PUFAs were unable to prevent progression to NASH. In contrast to the STZ/HFD mouse model, the other studies induced less progressive forms of NAFLD, which might be crucial for the overall outcome. These findings suggest that n-3 PUFAs hamper steatosis, but finally fail to prevent progression to more severe and complex forms of NAFLD. Hence, the effects described herein may help to understand the discrepancies between different studies.

In humans, NAFLD is often associated with the metabolic syndrome (including obesity and insulin resistance), which is incompletely reflected by the STZ/ HFD mouse model. In fact, these mice are lean and the elevated blood glucose levels caused by STZ-induced betacell death rather reflect a 'type I-like' diabetes. To date, the lack of metabolic determinants of the metabolic syndrome in mouse models of NAFLD is a commonly known problem, limiting most mouse models of progressive NAFLD/NASH to reflect the human situation. Nevertheless, the STZ/HFD mouse model is a commonly used model of NAFLD as liver disease develops progressively in a short period of time.

CD36 is a scavenger receptor which is involved in fatty acid uptake and signal transduction (29). Herein, we reported upregulated $C d 36$ mRNA expression in STZ/ HFD treated wt mice and significantly lower values in fat-1 transgenic STZ/HFD mice at 6 weeks of age but not at 8 weeks. However, mRNA expression of other genes involved in lipid uptake and transport (Fatp2, Ftp5, Fabp1) was not affected by STZ/HFD treatment at any examined time point. It is known that hepatic CD36 protein and mRNA expression is elevated in NAFLD patients (30) and positively correlates with the liver fat content (31). Similarly, increased $C d 36$ mRNA expression has been observed in mice with NASH (32). While mice protected from diet induced NAFLD display decreased CD36 (33) and PPAR- $\gamma$ expression, other genes involved in lipid metabolism and transport like FATP5 were not altered (34). Furthermore, it has been shown that $\mathrm{CD} 36$ deficient mice are protected from NAFLD and insulin resistance (35). Therefore, the rise of $C d 36$ mRNA expression in fat-1 STZ/HFD mice at 8 weeks might account for the lacking protection against $\mathrm{NASH}$ at that time point.

It is known that PPAR- $\gamma$ regulates $C d 36(34,36)$ and Plin2 (37) mRNA expression. Herein, we showed that hepatic Pparg mRNA expression was significantly reduced in fat-1 transgenic STZ/HFD mice compared to wt STZ/ HFD mice. However, PPAR- $\gamma$ expression is found to be increased in NAFLD (38), and PPAR- $\gamma$ polymorphisms are associated with NAFLD development and severity $(39,40)$. Accordingly, knock out of PPAR- $\gamma$ in hepatocytes or Kupffer cells has been shown to alleviate NAFLD in mice (41). Due to the role of PPAR- $\gamma$ in fatty acids transport and storage, its down regulation might contribute to reduced liver fat accumulation in fat- 1 transgenic STZ/HFD mice. By facilitating uptake of PPAR- $\gamma$ agonists like fatty acids, CD36 controls its own expression in a feed-forward cycle (42). Therefore, increased fatty acid supply leads to increased fatty acid uptake. This mechanism might account for the delayed increase of $C d 36$ mRNA expression in fat-1 STZ/HFD mice at 8 weeks. We suggest that, even though fatty acid uptake mechanisms were initially impaired in fat-1 STZ/HFD mice, continuous supply with fat and sustained insult of diabetes, still results in fatty acid delivery to the liver. Furthermore, beside protein facilitated fatty acid transport, fatty acid uptake by simple diffusion is possible (43). Therefore, upon continuous fatty acid oversupply passive diffusion might counteract regulatory mechanisms of protein assisted fatty acid transport.

Perilipins (Plin) are a protein family that is involved in lipid storage as coating proteins on lipid droplets. Herein, we observed increased levels of n-3 PUFAs reduce Plin2 and Plin 3 but not Plin 5 mRNA expression in 6 week old but not 8 week old STZ/HFD treated mice. It has been shown that Plin2 expression correlates with the degree of 
hepatic steatosis in human patients (44). Similarly, in an in vitro model of hepatic steatosis, cultured hepatocytes displayed increasing Plin 2 mRNA expression with rising degree of steatosis (45). Several knock down and knock out experiments have been conducted to reveal a causal link between Plin2 expression and NAFLD. Liver specific Plin2 knock out results in alleviated steatosis and inflammation in methionine choline deficient diet fed mice (46). Similar effects were obtained with Plin 2 knock down in HFD fed mice, resulting in improved steatosis and insulin resistance (47). Whereas in humans Plin 3 is expressed in non-steatotic as well as steatotic liver $(44,48)$, its expression is upregulated in mice upon HFD feeding (49). Of interest, a Plin 3 knock down improves steatosis, triglyceride content, glucose tolerance and insulin sensitivity in mice $(48,49)$. Thus, we suggest that down regulation of Plin2 and Plin 3 expression at 6 weeks in fat-1 STZ/HFD mice might contribute to the transient protective effects of n-3 PUFAs observed at this time point.

In summary, an increased n-3 PUFA level and n-3/n-6 ratio in liver tissue of fat-1 transgenic STZ/HFD mice just transiently improved NASH pathology, but failed to prevent NASH development. These effects might be caused by a short-term reduction of genes involved in fatty acid uptake and lipid storage in the early phase of NASH. Thus, n-3 PUFAs might help to hamper NAFLD when the underlying cause is eliminated. However, under a continuous insult, like fatty acid over-supply and increased blood glucose levels, fat-1 transgenic STZ/HFD mice are not protected from NASH.

\section{Acknowledgments}

We thank Dr. Karsten-Henrich Weylandt and Dr. Jing X. Kang who provided and gave permission for use of fat-1 mice, respectively. Furthermore, we thank Alexander Hartmann, Berit Blendow, Chantal von Hörsten, Dorothea Frenz, Eva Lorbeer, Ilona Klammfuß, Laura Grüner, Mareike Degner and Maren Nerowski for their excellent technical assistance. The authors wish to thank Birgit Jentz and Maria Dahm of the Institute of Muscle Biology and Growth who collaborated in terms of sample preparation and GC measurements.

Funding: This work was supported by the Deutsche Forschungsgemeinschaft, Bonn-Bad Godesberg, Germany (AB 453/2-1).

\section{Footnote}

Conflicts of Interest: The authors have no conflicts of interest to declare.

Ethical Statement: All animal experiments were approved by "Landesamt für Landwirtschaft, Lebensmittelsicherheit und Fischerei Mecklenburg-Vorpommern" (7221.3-1022/15) and were executed in accordance with the German legislation and EU-directive 2010/63/EU.

\section{References}

1. Younossi ZM. Patient-reported Outcomes and the Economic Effects of Non-Alcoholic Fatty Liver Disease and Non-alcoholic Steatohepatitis - The Value Proposition. Hepatology 2018;68:2405-12.

2. Estes C, Razavi H, Loomba R, et al. Modeling the epidemic of nonalcoholic fatty liver disease demonstrates an exponential increase in burden of disease. Hepatology 2018;67:123-33.

3. Wong VWS, Chitturi S, Wong GL, et al. Pathogenesis and novel treatment options for non-alcoholic steatohepatitis. Lancet Gastroenterol Hepatol 2016;1:56-67.

4. Simopoulos AP. The Importance of the Omega-6/ Omega-3 Fatty Acid Ratio in Cardiovascular Disease and Other Chronic Diseases. Exp Biol Med (Maywood) 2008;233:674-88.

5. Saini RK, Keum YS. Omega-3 and omega-6 polyunsaturated fatty acids: Dietary sources, metabolism, and significance - A review. Life Sci 2018;203:255-67.

6. Musa-Veloso K, Venditti C, Lee HY, et al. Systematic review and meta-analysis of controlled intervention studies on the effectiveness of long-chain omega- 3 fatty acids in patients with nonalcoholic fatty liver disease. Nutr Rev 2018;76:581-602.

7. Patterson E, Wall R, Fitzgerald GF, et al. Health Implications of High Dietary Omega-6 Polyunsaturated Fatty Acids. J Nutr Metab 2012;2012:539426.

8. Serhan CN. Novel Pro-Resolving Lipid Mediators in Inflammation Are Leads for Resolution Physiology. Nature 2014;510:92-101.

9. Marventano S, Kolacz P, Castellano S, et al. A review of recent evidence in human studies of n-3 and n-6 PUFA intake on cardiovascular disease, cancer, and depressive disorders: Does the ratio really matter? Int J Food Sci Nutr 2015;66:611-22. 
10. Serhan CN, Levy BD. Resolvins in inflammation: emergence of the pro-resolving superfamily of mediators. J Clin Invest 2018;128:2657-69.

11. Kang JX, Wang J, Wu L, et al. Fat-1 mice convert n-6 to n-3 fatty acids. Nature 2004;427:504-5.

12. Das UN, Puskás LG. Transgenic fat-1 mouse as a model to study the pathophysiology of cardiovascular, neurological and psychiatric disorders. Lipids Health Dis 2009;8:61.

13. Shin SC, Lee KM, Kang YM, et al. Alteration of cytokine profiles in mice exposed to chronic low-dose ionizing radiation. Biochem Biophys Res Commun 2010;397:644-9.

14. Fujii M, Shibazaki Y, Wakamatsu K, et al. A murine model for non-alcoholic steatohepatitis showing evidence of association between diabetes and hepatocellular carcinoma. Med Mol Morphol 2013;46:141-52.

15. Liebig $M$, Hassanzada A, Kämmerling $M$, et al. Microcirculatory disturbances and cellular changes during progression of hepatic steatosis to liver tumors. Exp Biol Med (Maywood) 2018;243:1-12.

16. Abshagen K, Mense L, Fischer F, et al. Repin 1 deficiency in liver tissue alleviates NAFLD progression in mice. J Adv Res 2018;16:99-111.

17. Kleiner DE, Brunt EM, Van Natta M, et al. Design and validation of a histological scoring system for nonalcoholic fatty liver disease. Hepatology 2005;41:1313-21.

18. Abshagen K, Eipel C, Kalff JC, et al. Loss of NF-kappaB activation in Kupffer cell-depleted mice impairs liver regeneration after partial hepatectomy. Am J Physiol Gastrointest Liver Physiol 2007;292:G1570-7.

19. Dannenberger D, Nuernberg K, Nuernberg G, et al. Different dietary protein and PUFA interventions alter the fatty acid concentrations, but not the meat quality, of porcine muscle. Nutrients 2012;4:1237-46.

20. Dannenberger D, Nuernberg G, Nuernberg K, et al. Effects of diets supplemented with n-3 or n-6 PUFA on pig muscle lipid metabolites measured by non-targeted LC-MS lipidomic profiling. J Food Compos Anal 2017;56:47-54.

21. Parker HM, Johnson NA, Burdon CA, et al. Omega-3 supplementation and non-alcoholic fatty liver disease: A systematic review and meta-analysis. J Hepatol 2012;56:944-51.

22. Yan JH, Guan BJ, Gao HY, et al. Omega-3 polyunsaturated fatty acid supplementation and non-alcoholic fatty liver disease. Medicine (Baltimore) 2018;97:e12271.

23. Argo CK, Patrie JT, Lackner C, et al. Effects of N-3 Fish Oil on Metabolic and Histological Parameters in NASH: A Double-Blind, Randomized, Placebo-Controlled Trial. J Hepatol 2015;62:190-7.
24. Sanyal AJ, Abdelmalek MF, Suzuki A, et al. No significant effects of ethyl-eicosapentanoic acid on histologic features of nonalcoholic steatohepatitis in a phase 2 triaL. Gastroenterology 2014;147:377-84.e1.

25. Provenzano A, Milani S, Vizzutti F, et al. N-3 polyunsaturated fatty acids worsen inflammation and fibrosis in experimental nonalcoholic steatohepatitis. Liver Int 2014;34:918-30.

26. Bae JS, Park JM, Lee J, et al. Amelioration of nonalcoholic fatty liver disease with NPC1L1-targeted IgY or $\mathrm{n}-3$ polyunsaturated fatty acids in mice. Metabolism 2017;66:32-44.

27. Kim EH, Bae JS, Hahm KB, et al. Endogenously synthesized $n-3$ polyunsaturated fatty acids in fat- 1 mice ameliorate high-fat diet-induced non-alcoholic fatty liver disease. Biochem Pharmacol 2012;84:1359-65.

28. Guo XF, Gao J, Li J, et al. Fat-1 Mice Prevent High-Fat Plus High-Sugar Diet-Induced Non-Alcoholic Fatty Liver Disease. Food Funct 2017;8:4053-61.

29. Pepino MY, Kuda O, Samovski D, et al. StructureFunction of CD36 and Importance of Fatty Acid Signal Transduction in Fat Metabolism. Annu Rev Nutr 2014;34:281-303.

30. Miquilena-Colina ME, Lima-Cabello E, Sánchez-Campos $\mathrm{S}$, et al. Hepatic fatty acid translocase CD36 upregulation is associated with insulin resistance, hyperinsulinaemia and increased steatosis in non-alcoholic steatohepatitis and chronic hepatitis C. Gut 2011;60:1394-402.

31. Greco D, Kotronen A, Westerbacka J, et al. Gene expression in human NAFLD. Am J Physiol Gastrointest Liver Physiol 2008;294:G1281-7.

32. Dorn C, Engelmann JC, Saugspier M, et al. Increased expression of c-Jun in nonalcoholic fatty liver disease. Lab Investig 2014;94:394-408.

33. Niu B, He K, Li P, et al. SIRT1 upregulation protects against liver injury induced by a HFD through inhibiting CD36 and the NF- $\kappa$ B pathway in mouse kupffer cells. Mol Med Rep 2018;18:1609-15.

34. Liu Y, Cheng F, Luo Y, et al. PEGylated Curcumin Derivative Attenuates Hepatic Steatosis via CREB/PPAR- $\gamma /$ CD36 Pathway. Biomed Res Int 2017;2017:8234507.

35. Wilson CG, Tran JL, Erion DM, et al. Hepatocyte-specific disruption of CD36 attenuates fatty liver and improves insulin sensitivity in HFD-fed mice. Endocrinology 2016;157:570-85.

36. Lim HJ, Lee S, Lee KS, et al. PPAR $\gamma$ activation induces CD36 expression and stimulates foam cell like changes 
in rVSMCs. Prostaglandins Other Lipid Mediat 2006;80:165-74.

37. Okumura T. Role of lipid droplet proteins in liver steatosis. J Physiol Biochem 2011;67:629-36.

38. Pettinelli P, Videla LA. Up-regulation of PPAR- $\gamma$ mRNA expression in the liver of obese patients: An additional reinforcing lipogenic mechanism to SREBP-1c induction. J Clin Endocrinol Metab 2011;96:1424-30.

39. Yang Z, Wen J, Li Q, et al. PPARG gene Pro12Ala variant contributes to the development of non-alcoholic fatty liver in middle-aged and older Chinese population. Mol Cell Endocrinol 2012;348:255-9.

40. Gawrieh S, Marion MC, Komorowski R, et al. Genetic variation in the peroxisome proliferator activated receptorgamma gene is associated with histologically advanced NAFLD. Dig Dis Sci 2012;57:952-7.

41. Morán-Salvador E, Lopez-Parra M, Garcia-Alonso V, et al. Role for PPAR in obesity-induced hepatic steatosis as determined by hepatocyte- and macrophage-specific conditional knockouts. FASEB J 2011;25:2538-50.

42. Glatz JFC, Luiken JJFP. Dynamic role of the transmembrane glycoprotein CD36 (SR-B2) in cellular fatty acid uptake and utilization. J Lipid Res 2018;59:1084-93.

Cite this article as: Liebig M, Dannenberger D, Vollmar B, Abshagen K. Endogenously increased n-3 PUFA levels in fat-1 transgenic mice do not protect from non-alcoholic steatohepatitis. HepatoBiliary Surg Nutr 2019;8(5):447-458. doi: $10.21037 /$ hbsn.2019.04.03
43. Hamilton JA, Guo W, Kamp F. Mechanism of cellular uptake of long-chain fatty acids: Do we need cellular proteins? Mol Cell Biochem 2002;239:17-23.

44. Straub BK, Stoeffel P, Heid H, et al. Differential pattern of lipid droplet-associated proteins and de novo perilipin expression in hepatocyte steatogenesis. Hepatology 2008;47:1936-46.

45. Grasselli E, Voci A, Pesce C, et al. PAT protein mRNA expression in primary rat hepatocytes: Effects of exposure to fatty acids. Int J Mol Med 2010;25:505-12.

46. Najt CP, Senthivinayagam S, Aljazi MB, et al. Liverspecific loss of Perilipin 2 alleviates diet-induced hepatic steatosis, inflammation, and fibrosis. Am J Physiol Gastrointest Liver Physiol 2016;310:G726-38.

47. Varela GM, Antwi DA, Dhir R, et al. Inhibition of ADRP prevents diet-induced insulin resistance. Am J Physiol Gastrointest Liver Physiol 2008;295:G621-8.

48. Carr RM, Ahima RS. Pathophysiology of lipid droplet proteins in liver diseases. Exp Cell Res 2016;340:187-92.

49. Carr RM, Patel RT, Rao V, et al. Reduction of TIP47 improves hepatic steatosis and glucose homeostasis in mice. Am J Physiol Regul Integr Comp Physiol 2012;302:R996-1003. 